\title{
Identification of Chili Pepper Genotypes (Capsicum spp.) Resistant to Meloidogyne enterolobii
}

\author{
M. L. S. Marques ${ }^{1}$, J. V. G. Chadud ${ }^{2}$, M. F. Oliveira ${ }^{2}$, A. R. Nascimento ${ }^{2} \&$ M. R. Rocha ${ }^{2}$ \\ ${ }^{1}$ Instituto Federal Goiano, Campus Ceres, Goias, Brazil \\ ${ }^{2}$ Escola de Agronomia, Universidade Federal de Goiás, Goiás, Brazil \\ Correspondence: Mara Rubia da Rocha, Universidade Federal de Goias, Escola de Agronomia, Avenida \\ Esperança, s/n, Campus Samambaia, CEP 74690-900, Goiânia, Goiás, Brazil. Tel: 55-629-9687-1878. E-mail: \\ darochamararubia@gmail.com
}

Received: February 14, 2019 Accepted: April 6, $2019 \quad$ Online Published: June 15, 2019

doi:10.5539/jas.v11n8p165 URL: https://doi.org/10.5539/jas.v11n8p165

The research was financed by FAPEG (Fundação de Amparo à Pesquisa do Estado de Goiás).

\begin{abstract}
Chili pepper has economic importance and is the dominant Solanaceae in the market of spicy spices. Among the pathogens that affect this crop, Meloidogyne enterolobii is one of the most important because it presents a wide range of hosts and there are no resistance genes identified that are efficient against this species. The present study aimed to evaluate the reaction of chili pepper genotypes (Capsicum spp.) to M. enterolobii in order to identifify genetic resistance. Three experiments were conducted in a completely randomized design under greenhouse conditions: Experiment I, with 53 genotypes with ten replications; Experiment II, with twenty genotypes with ten replications; Experiment III in a $16 \times 4$ factorial scheme, with sixteen Capsicum spp. genotypes and four inoculum concentrations of $M$. enterolobii and eight replications. Inoculation was performed seven days after transplanting the Capsicum spp. seedlings into 2L plastic bags filled with sterilized soil and sand (2:1), using 4,000 eggs + J2/plant. Ninety days after inoculation (DAI) (Experiments I and II) and 60 DAI (Experiment III) nematode population density, reproduction factor (RF), fresh root mass, egg mass index (EMI) and gall index (GI) were evaluated. Genotypes with RF $<1.0$ were considered resistant according to Oostenbrink (1966). Thirty one genotypes of Capsicum spp. showed resistance to M. enterolobii with RF ranging from 0.87 to 0.08 . Seventeen resistant genotypes of $C$. chinensis presented RF lower than 0.85 , ten genotypes of $C$. annuum had the RF lower than 0.75 , three genotypes of $C$. frutescens had the RF lower than 0.87 and only one genotype of $C$. baccatum was resistant to M. enterolobii, presenting RF $=0.6$. EMI and GI weren't considered reliable variables to determine resistance and susceptibility. Fourteen genotypes rated as resistant in Experiments I and II were submitted to increasing concentrations of inoculum and, nevertheless, remained resistant.
\end{abstract}

Keywords: root-knot nematode, genetic resistance, Capsicum chinensis, C. annuum, C. baccatum, C. frutescens

\section{Introduction}

The species of pepper of the genus Capsicum originate in the Americas and have been consumed for more than $7,000,000$ years. They are grown around the world, with the largest producers being China, Thailand, South Korea, India, Japan, Mexico, United States, Brazil and Argentina (Rufino \& Penteado, 2006; Pinto et al., 2011). In Brazil around five thousand hectares of sweet and chili peppers are cultivated producing 75 thousand tons (Ribeiro et al., 2008). The main Brazilian producing states are Minas Gerais, Goiás, São Paulo, Ceará and Rio Grande do Sul (Costa \& Henz, 2007).

According to Reifschneider (2000), the species of Capsicum are classified into 33 domesticated, wild and semi-domesticated species. The domesticated species are represented by: Capsicum annuum L., Capsicum chinenses Jacq, Capsicum frutescens L., Capsicum baccatum L. and Capsicum pubescens Ruiz and Pavon, the latter not being present in Brazil. The genus Capsicum spp. belongs to the Solanaceae family and presents wide genetic variability regarding its shape, size, color, fruit flavor and pungency.

Species of the Solanaceae family, especially chili pepper and sweet pepper (Capsicum spp.) and tomato (Solanum lycopersicum L.) are the most cultivated vegetables in the world and are highly susceptible to the 
root-knot nematodes (Meloidogyne sp.). The genetic resistance to these nematodes is one of the most efficient, economical and with the least environment impact forms of control (Carneiro et al., 2006). The use of tomato and pepper cultivars with resistance to M. incognita, M. javanica and M. arenaria is well established. These cultivars carry the Mi gene (Sasser \& Kirby, 1979; Fargette, 1987). However, the species M. enterolobii has been detected parasitizing tomato plants and peppers resistant to M. incognita, M. arenaria and M. javanica (Carneiro et al., 2006).

The ability of $M$. enterolobii to parasitize plants resistant to other species of Meloidogyne makes this plant nematode extremely important for national agriculture. The knowledge of hosts is a fundamental step for the adoption of control strategies that prevent infestations of new areas (Bitencourt \& Silva, 2010). In view of the increasingly frequent reports of the occurrence of $M$. enterolobii and the absence of commercial cultivars of pepper and tomato resistant to this nematode, it is necessary to search for Capsicum genotypes with resistance to this species. Thus, this study had the objective to evaluate the reaction of pepper genotypes (Capsicum spp.) to $M$. enterolobii aiming identification of genetic resistance.

\section{Method}

The M. enterolobii population used in this study was obtained in the field from roots of guava (Psidium guajava L.) cv. Paluma. This population was isolated and multiplied in tomato plants (Solanum lycopersicum L.) cv. Santa Cruz Kada. The confirmation of the Meloidogyne species was made using the isoenzyme electrophoresis technique described by Carneiro and Almeida (2001)

Three experiments were conducted under greenhouse conditions (geographic coordinates $16^{\circ} 40^{\prime} 22^{\prime \prime} \mathrm{S}$ and $49^{\circ} 15^{\prime} 19^{\prime \prime}$ W). Experiments I and II were performed from February to April/2015 and February to April/2016, respectively, to evaluate the hostability of 73 pepper genotypes to M. enterolobii. Experiment III was conducted from February to May/2016 submitting selected genotypes to increasing inoculum concentrations.

Experiments I and II were carried out in a completely randomized design with ten replicates. Experiment I had 53 treatments and Experiment II had 20 treatments. The treatments consisted of chili pepper genotypes (Capsicum spp.) obtained from the UFG seed collection. The tomato cv. Santa Cruz Kada was used as susceptible standard in all experiments to verify the inoculum viability.

Seeding was done in plastic seedling trays with 400 inverted pyramidal cells, filled with $40 \mathrm{~cm}^{3}$ of commercial substrate Bioplant ${ }^{\circledR}$ (Bioplant Agrícola Ltda., Nova Ponte, MG) per cell. After 30 days, when the seedlings presented two to four pairs of leaves, they were transplanted into black polyethylene bags with capacity for two liters, filled with substrate (sand: soil) previously autoclaved $\left(120^{\circ} \mathrm{C}\right.$ for 2 hours) in a ratio of 2:1.

After seven days of transplanting of the seedlings, artificial inoculation was performed, with $4 \mathrm{~mL}$ of aqueous suspension containing approximately 4,000 eggs $+\mathrm{J} 2$ of $M$. enterolobii per plot (one plant/bag). This was considered as the initial nematode population (Ip). During the period of experiment conduction, daily watering was performed.

The evaluations were performed at 90 days after inoculation (DAI). The plants were removed from the plastic bags, the shoots were discarded and the roots washed under running water and allowed to dry on paper towels. The roots were weighed on a digital scale $(0.01 \mathrm{~g}$ precision) and then stained with Floxin B $(0.015 \%)$, allowed to stand for 15-20 minutes and then washed with tap water to remove the residual dye from the roots. The egg masses external to the roots were colored, facilitating the visualization and counting under a stereoscopic microscope. Gall index (GI) and egg mass index (EMI) were obtained after counting, according to Taylor and Sasser (1978).

The roots were then processed for nematode extraction, being cut into fragments of approximately $2 \mathrm{~cm}$ in length and ground in a blender containing $0.5 \% \mathrm{NaClO}$ solution. The suspension obtained was poured onto a 100 mesh ( $0.149 \mathrm{~mm}$ aperture) sieve coupled onto a 500 mesh $(0.025 \mathrm{~mm}$ aperture $)$ sieve. The material trapped in the 500 mesh screen was carefully washed under running water to remove the excess $\mathrm{NaClO}$ then collected into a beaker. The suspension was then transferred to centrifuge tubes, calibrated and $1 \mathrm{~mL}$ of kaolin was added to each tube and homogenized with a glass rod. The samples were centrifuged for 5 minutes at 1800 RPM and then the supernatant was discarded. The sucrose solution $(0.454 \mathrm{Kg} / \mathrm{L})$ was added to the pellet and homogenized. The samples were centrifuged for 1 minute at 1800 RPM and then the supernatant was poured into the 500 mesh sieve, washed thoroughly with tap water to remove the excess sucrose (Coolen \& D'Herde, 1972). After extraction, the nematodes were collected and reserved in $50 \mathrm{~mL}$ test tubes for further counting under an optical microscope (40× magnification) with the aid of a Peters slide. After counting the nematodes, the population density was obtained and expressed in number of eggs and $\mathrm{J} 2 / 10 \mathrm{~g}$ of root. 
The total number of nematodes in each root system was considered the final population (Fp). The reproduction factor (RF) was obtained by the ratio between the final population $(\mathrm{Fp})$ and the initial population (Ip) $(\mathrm{RF}=$ $\mathrm{Fp} / \mathrm{Ip}$ ). The plants were then classified as resistant or susceptible to M. enterolobii, according to Oostenbrink criterion (1966), being considered resistant those with $\mathrm{RF}<1.0$ and susceptible those with $\mathrm{RF}>1.0$.

For experiment III, we selected sixteen genotypes of Capsicum spp. that were evaluated in Experiment I: fourteen resistant and two susceptible (UFGCBA 03-Cambuci and UFGCCH 02-Sete Molhos) to M. enterolobii. The genotypes were submitted to increasing inoculum concentrations of $M$. enterolobii $(0,2,000,4,000$ and 8,000 eggs $+\mathrm{J} 2 /$ plant) using a completely randomized experimental design (DIC) in a $16 \times 4$ factorial scheme (Capsicum spp. genotypes $\times$ inoculum concentrations) with eight replications.

The seedlings were obtained according to the methodology previously described. When the seedlings presented two to four pairs of leaves (30 days after sowing), they were transplanted into $2 \mathrm{~L}$ black polyethylene bags containing substrate (sand and soil at rate $2: 1$ ) previously sterilized at $120^{\circ} \mathrm{C}$ for 2 hours.

The inoculation with $M$. enterolobii at the concentrations corresponding to the treatments was performed after 15 days of transplanting. At 60 DAI the roots were submitted to evaluations of egg mass index (EMI), gall index (GI), fresh root mass (FRM), nematode population density (Pd) and the reproduction factor (RF) as described above.

The data were submitted to the Lillieford and Bartlett test (Conagin et al., 1993) for continuous and discrete quantitative dependent variables to test for normality and homoscedasticity. When necessary the data were transformed.

The data of Experiments I, II and III were submitted to analysis of variance, and the means were compared by the Scott-Knott test $(p<0.05)$ using the Assistat version 7.7 software (Silva, 2017). For the 73 genotypes (Experiments I and II) the multivariate approach was applied through principal component analysis (correlation path) and clustering technique (Hair Junior et al., 2005). The dendogram was obtained by the UPGMA method using the Bray-Curtis distance as a similarity coefficient using the Past software version 3.18 (Hammer et al., 2001). For Experiment III, the analysis of variance was made considering the factorial scheme. When a significant interaction between genotypes and inoculum concentration was observed, regression analysis was performed using the Sisvar software (Ferreira, 2011).

\section{Results}

Of the 73 genotypes of Capsicum spp. evaluated in Experiments I and II, 31 presented resistance to $M$. enterolobii, according to Oostenbrink (1966) criteria. Among the resistant genotypes, seventeen are C. chinensis, ten are C. annum, one C. baccatum and three C. frutescens (Table 1). 
Table 1. Reaction of 73 Capsicum spp. genotypes to M. enterolobii, under greenhouse conditions, at 90 days after inoculation. UFG, GO, 2017

\begin{tabular}{|c|c|c|c|c|c|c|c|c|c|c|c|}
\hline \multicolumn{12}{|l|}{ Experiment I } \\
\hline \multirow{2}{*}{$\begin{array}{l}\text { Genotypes } \\
\text { Capsicum chinenses }\end{array}$} & \multirow[t]{2}{*}{ Ident. UFG } & \multicolumn{2}{|l|}{ FRM $^{1}$} & \multicolumn{2}{|l|}{$\mathbf{P d}^{2}$} & \multirow[t]{2}{*}{$\mathbf{R F}^{3}$} & \multicolumn{2}{|l|}{$\mathbf{G I}^{4}$} & \multicolumn{2}{|l|}{$\mathrm{EMI}^{5}$} & \multirow[t]{2}{*}{$\mathbf{R}^{6}$} \\
\hline & & & & & & & & & & & \\
\hline Naga Jalokia A & UFGCCH 01 & 15.73 & d & 42492 & $\mathrm{~b}$ & 14.89 & 4.75 & $\mathrm{a}$ & 4.62 & $\mathrm{a}$ & S \\
\hline Sete Molhos & UFGCCH 02 & 24.05 & $\mathrm{~b}$ & 20142 & $\mathrm{c}$ & 11.46 & 3.87 & $\mathrm{~b}$ & 3.87 & $\mathrm{~b}$ & $\mathrm{~S}$ \\
\hline Bico de Papagaio & UFGCCH 03 & 15.91 & $\mathrm{~d}$ & 23702 & $\mathrm{c}$ & 8.05 & 4.87 & $\mathrm{a}$ & 4.87 & $\mathrm{a}$ & $\mathrm{S}$ \\
\hline Chifre de Veado AR2 & UFGCCH 04 & 20.44 & $\mathrm{c}$ & 12727 & $\mathrm{~d}$ & 7.18 & 4.12 & $\mathrm{a}$ & 3.87 & $\mathrm{~b}$ & $\mathrm{~S}$ \\
\hline Amazoninha Amarela & UFGCCH 05 & 13.55 & $\mathrm{~d}$ & 11885 & $\mathrm{~d}$ & 4.51 & 5.00 & $\mathrm{a}$ & 5.00 & $\mathrm{a}$ & S \\
\hline Pimenta Cheiro Vermelha & UFGCCH 06 & 29.20 & $\mathrm{~b}$ & 4987 & $\mathrm{~d}$ & 3.06 & 3.00 & $\mathrm{~b}$ & 2.75 & $\mathrm{~b}$ & $\mathrm{~S}$ \\
\hline Biquinho A & UFGCCH 07 & 22.26 & $\mathrm{c}$ & 7035 & $\mathrm{~d}$ & 2.91 & 4.62 & $\mathrm{a}$ & 4.25 & $\mathrm{a}$ & $\mathrm{S}$ \\
\hline Pimenta de Cheiro Amarela & UFGCCH 08 & 32.82 & $\mathrm{a}$ & 2802 & $\mathrm{e}$ & 2.20 & 3.00 & $\mathrm{~b}$ & 2.87 & $\mathrm{~b}$ & $\mathrm{~S}$ \\
\hline Bode Roxa B & UFGCCH 09 & 21.86 & $\mathrm{c}$ & 2506 & e & 1.32 & 1.50 & $\mathrm{c}$ & 0.50 & $\mathrm{~d}$ & $\mathrm{~S}$ \\
\hline Fidalga Amarela A & UFGCCH 10 & 14.33 & $\mathrm{~d}$ & 1180 & $\mathrm{e}$ & 0.55 & 0.62 & $\mathrm{~d}$ & 1.00 & $\mathrm{c}$ & $\mathrm{R}$ \\
\hline Bode Vermelha B & UFGCCH 11 & 15.33 & $\mathrm{~d}$ & 1546 & $\mathrm{e}$ & 0.55 & 3.62 & $\mathrm{~b}$ & 3.62 & $\mathrm{~b}$ & $\mathrm{R}$ \\
\hline Bode Vermelha A & UFGCCH 12 & 34.48 & $\mathrm{a}$ & 668 & $\mathrm{e}$ & 0.53 & 4.37 & $\mathrm{a}$ & 4.37 & a & $\mathrm{R}$ \\
\hline Habanero Amarela A & UFGCCH 13 & 21.30 & $\mathrm{c}$ & 753 & $\mathrm{e}$ & 0.47 & 2.87 & $\mathrm{~b}$ & 3.50 & $\mathrm{~b}$ & $\mathrm{R}$ \\
\hline Chifre de Veado C & UFGCCH 14 & 19.64 & $\mathrm{c}$ & 680 & $\mathrm{e}$ & 0.40 & 1.37 & $\mathrm{c}$ & 1.12 & $\mathrm{c}$ & $\mathrm{R}$ \\
\hline Redonda de Vaso Vermelha & UFGCCH 15 & 20.57 & $\mathrm{c}$ & 740 & $\mathrm{e}$ & 0.39 & 3.62 & $\mathrm{~b}$ & 3.62 & $\mathrm{~b}$ & $\mathrm{R}$ \\
\hline Bode Amarela & UFGCCH 16 & 22.73 & $\mathrm{c}$ & 745 & $\mathrm{e}$ & 0.38 & 1.62 & $\mathrm{c}$ & 1.62 & $\mathrm{c}$ & $\mathrm{R}$ \\
\hline Murupi & UFGCCH 17 & 18.18 & $\mathrm{c}$ & 716 & $\mathrm{e}$ & 0.37 & 0.62 & $\mathrm{~d}$ & 0.62 & $\mathrm{~d}$ & $\mathrm{R}$ \\
\hline Pirâmide de Vaso & UFGCCH 18 & 16.59 & $\mathrm{~d}$ & 1322 & $\mathrm{e}$ & 0.36 & 2.87 & $\mathrm{~b}$ & 3.12 & $\mathrm{~b}$ & $\mathrm{R}$ \\
\hline Vulcão Feltrin & UFGCCH 19 & 20.51 & $\mathrm{c}$ & 557 & e & 0.32 & 0.12 & $\mathrm{~d}$ & 0.12 & $\mathrm{~d}$ & $\mathrm{R}$ \\
\hline Espanhola & UFGCCH 20 & 33.25 & a & 391 & $\mathrm{e}$ & 0.30 & 1.50 & $\mathrm{c}$ & 1.37 & $\mathrm{c}$ & $\mathrm{R}$ \\
\hline Pitanga & UFGCCH 21 & 22.56 & $\mathrm{c}$ & 531 & $\mathrm{e}$ & 0.28 & 4.12 & $\mathrm{a}$ & 4.37 & $\mathrm{a}$ & $\mathrm{R}$ \\
\hline Redonda de Vaso Amarela & UFGCCH 22 & 27.97 & $\mathrm{~b}$ & 649 & e & 0.23 & 1.75 & $\mathrm{c}$ & 1.37 & $\mathrm{c}$ & $\mathrm{R}$ \\
\hline Cheiro de Baiana & UFGCCH 23 & 15.61 & $\mathrm{~d}$ & 464 & $\mathrm{e}$ & 0.19 & 1.00 & $\mathrm{~d}$ & 1.00 & $\mathrm{~d}$ & $\mathrm{R}$ \\
\hline Bode Roxa A & UFGCCH 24 & 15.70 & $\mathrm{~d}$ & 745 & $\mathrm{e}$ & 0.08 & 0.00 & $\mathrm{~d}$ & 1.50 & $\mathrm{c}$ & $\mathrm{R}$ \\
\hline \multicolumn{12}{|l|}{ Capsicum annuum } \\
\hline Páprica 96L & UFGCA 01 & 22.22 & $\mathrm{c}$ & 51109 & $\mathrm{~b}$ & 23.61 & 4.62 & $\mathrm{a}$ & 4.62 & $\mathrm{a}$ & S \\
\hline Carine Feltrin A & UFGCA 02 & 8.54 & d & 158784 & $\mathrm{a}$ & 16.92 & 3.62 & $\mathrm{~b}$ & 3.87 & $\mathrm{~b}$ & $\mathrm{~S}$ \\
\hline Cumari Passarinho & UFGCA 03 & 21.40 & $\mathrm{c}$ & 25683 & c & 12.99 & 4.37 & $\mathrm{a}$ & 4.62 & $\mathrm{a}$ & $\mathrm{S}$ \\
\hline Cumari Amarela Pará B & UFGCA 04 & 5.68 & d & 78282 & $\mathrm{~b}$ & 7.36 & 2.00 & $\mathrm{c}$ & 2.12 & $\mathrm{c}$ & S \\
\hline Cumari B & UFGCA 05 & 29.93 & $\mathrm{~b}$ & 38335 & $\mathrm{c}$ & 5.67 & 3.87 & $\mathrm{~b}$ & 3.87 & $\mathrm{~b}$ & $\mathrm{~S}$ \\
\hline Páprica 541F & UFGCA 06 & 15.59 & $\mathrm{~d}$ & 11858 & $\mathrm{~d}$ & 4.74 & 4.87 & $\mathrm{a}$ & 5.00 & $\mathrm{a}$ & $\mathrm{S}$ \\
\hline Jalapeño A & UFGCA 07 & 33.79 & $\mathrm{a}$ & 7977 & $d$ & 3.92 & 4.75 & a & 4.62 & $\mathrm{a}$ & $\mathrm{S}$ \\
\hline Indiana A & UFGCA 08 & 20.16 & $\mathrm{c}$ & 7032 & $\mathrm{~d}$ & 3.33 & 0.25 & $\mathrm{~d}$ & 0.25 & $\mathrm{~d}$ & $\mathrm{~S}$ \\
\hline Cumari A & UFGCA 09 & 30.72 & $\mathrm{~b}$ & 1925 & $\mathrm{e}$ & 1.53 & 4.25 & $\mathrm{a}$ & 3.87 & $\mathrm{a}$ & $\mathrm{S}$ \\
\hline Carine Feltrin B & UFGCA 10 & 9.98 & d & 3503 & e & 1.12 & 3.75 & $\mathrm{~b}$ & 3.62 & $\mathrm{~b}$ & $\mathrm{~S}$ \\
\hline Cumari Cheirosa & UFGCA 11 & 15.76 & d & 1023 & $\mathrm{e}$ & 0.53 & 4.62 & $\mathrm{a}$ & 4.37 & $\mathrm{a}$ & $\mathrm{R}$ \\
\hline Cumari Vermelha & UFGCA 12 & 28.62 & $\mathrm{~b}$ & 523 & e & 0.47 & 3.87 & $\mathrm{~b}$ & 3.75 & $\mathrm{~b}$ & $\mathrm{R}$ \\
\hline Páprica Queen & UFGCA 13 & 19.41 & $\mathrm{c}$ & 979 & $\mathrm{e}$ & 0.39 & 3.62 & $\mathrm{~b}$ & 3.75 & $\mathrm{~b}$ & $\mathrm{R}$ \\
\hline Cayenne & UFGCA 14 & 14.55 & d & 2643 & $\mathrm{e}$ & 0.37 & 3.00 & $\mathrm{~b}$ & 2.87 & $\mathrm{~b}$ & $\mathrm{R}$ \\
\hline La Bombonera A & UFGCA 15 & 11.10 & d & 1308 & $\mathrm{e}$ & 0.36 & 4.50 & $\mathrm{a}$ & 4.50 & $\mathrm{a}$ & $\mathrm{R}$ \\
\hline Pimenta Comprida & UFGCA 16 & 11.96 & $\mathrm{~d}$ & 1439 & e & 0.35 & 3.62 & $\mathrm{~b}$ & 3.75 & $\mathrm{~b}$ & $\mathrm{R}$ \\
\hline Cumari C & UFGCA 17 & 33.54 & $\mathrm{a}$ & 425 & $\mathrm{e}$ & 0.35 & 0.12 & $\mathrm{~d}$ & 0.12 & d & $\mathrm{R}$ \\
\hline Cumari D & UFGCA 18 & 40.44 & $\mathrm{a}$ & 60 & $\mathrm{e}$ & 0.12 & 1.00 & $\mathrm{c}$ & 0.50 & $\mathrm{~d}$ & $\mathrm{R}$ \\
\hline \multicolumn{12}{|l|}{ Capsicum baccatum } \\
\hline Coração de Galinha & UFGCBA 01 & 19.28 & $\mathrm{c}$ & 71110 & $\mathrm{a}$ & 31.67 & 4.37 & $\mathrm{a}$ & 4.50 & $\mathrm{a}$ & $\mathrm{S}$ \\
\hline Dedo de Moça Vermelha & UFGCBA 02 & 22.80 & $\mathrm{c}$ & 16894 & $\mathrm{c}$ & 7.50 & 5.00 & a & 5.00 & $\mathrm{a}$ & $\mathrm{S}$ \\
\hline Cambuci & UFGCBA 03 & 25.09 & $\mathrm{~b}$ & 10899 & $\mathrm{~d}$ & 7.21 & 4.75 & $\mathrm{a}$ & 5.00 & a & $\mathrm{S}$ \\
\hline Tabasco & UFGCBA 04 & 13.83 & $\mathrm{~d}$ & 18885 & $\mathrm{~d}$ & 4.04 & 4.37 & $\mathrm{a}$ & 4.50 & $\mathrm{a}$ & $\mathrm{S}$ \\
\hline Dedo de Moça Amarela & UFGCBA 05 & 30.71 & $\mathrm{~b}$ & 4443 & $\mathrm{~d}$ & 3.11 & 4.12 & $\mathrm{a}$ & 4.12 & a & $\mathrm{S}$ \\
\hline Dedo de Moça & UFGCBA 06 & 13.71 & d & 2637 & e & 1.01 & 4.00 & $\mathrm{a}$ & 4.00 & a & $\mathrm{S}$ \\
\hline \multicolumn{12}{|l|}{ Capsicum frutescens } \\
\hline Vaso Picante Super Precoce A & UFGFR 01 & 18.91 & $\mathrm{c}$ & 5606 & e & 3.36 & 0.62 & d & 0.75 & $\mathrm{~d}$ & $\mathrm{~S}$ \\
\hline \multicolumn{12}{|l|}{ Capsicum frutescens } \\
\hline Malaguetinha & UFGFR 02 & 17.78 & $\mathrm{c}$ & 4624 & $\mathrm{e}$ & 1.88 & 1.25 & $\mathrm{c}$ & 1.37 & $\mathrm{c}$ & $\mathrm{S}$ \\
\hline Malagueta & UFGFR 03 & 27.25 & $\mathrm{~b}$ & 1022 & $\mathrm{e}$ & 0.87 & 3.75 & $\mathrm{~b}$ & 3.50 & $\mathrm{~b}$ & $\mathrm{R}$ \\
\hline Malaguetão & UFGFR 04 & 17.07 & $\mathrm{~d}$ & 1650 & $\mathrm{e}$ & 0.56 & 1.50 & $\mathrm{c}$ & 1.37 & $\mathrm{c}$ & $\mathrm{R}$ \\
\hline Vaso Picante Super Precoce B & UFGFR 05 & 24.37 & b & 937 & e & 0.53 & 3.25 & $\mathrm{~b}$ & 3.25 & $\mathrm{~b}$ & $\mathrm{R}$ \\
\hline $\mathrm{CV}(\%)$ & & 42.96 & & 95.44 & & 47.35 & 17.05 & & 17.30 & & \\
\hline
\end{tabular}




\begin{tabular}{|c|c|c|c|c|c|c|c|c|c|c|c|}
\hline \multicolumn{12}{|l|}{ Experiment II } \\
\hline \multirow{2}{*}{$\begin{array}{l}\text { Genotypes } \\
\text { Capsicum chinenses }\end{array}$} & \multirow[t]{2}{*}{ Ident. UFG } & \multicolumn{2}{|l|}{ FRM } & \multirow[t]{2}{*}{ Pd } & \multicolumn{2}{|c|}{ RF } & \multicolumn{2}{|l|}{ GI } & \multicolumn{2}{|l|}{ EMI } & \multirow[t]{2}{*}{$\mathbf{R}$} \\
\hline & & & & & & & & & & & \\
\hline Naga Jolokia B & UFGCCH 25 & 5.92 & a & 27446 & $\mathrm{a}$ & 3.63 & 5.00 & a & 5.00 & $a^{1}$ & $\mathrm{~S}$ \\
\hline Biquinho B & UFGCCH 26 & 2.62 & $\mathrm{~d}$ & 45940 & $\mathrm{a}$ & 1.99 & 4.50 & a & 4.50 & $\mathrm{a}$ & S \\
\hline Bode Vermelho C & UFGCCH 27 & 3.28 & $\mathrm{c}$ & 25107 & a & 1.86 & 3.80 & a & 3.80 & a & $\mathrm{S}$ \\
\hline Chifre de Veado C & UFGCCH 28 & 3.48 & c & 29920 & $\mathrm{a}$ & 1.84 & 4.50 & $\mathrm{a}$ & 4.50 & $\mathrm{a}$ & $\mathrm{S}$ \\
\hline Habanero Amarela B & UFGCCH 29 & 3.96 & $\mathrm{c}$ & 19846 & $\mathrm{~b}$ & 1.75 & 4.70 & $\mathrm{a}$ & 4.70 & $\mathrm{a}$ & $\mathrm{S}$ \\
\hline Cheiro Feltrin & UFGCCH 30 & 4.20 & $\mathrm{~b}$ & 19209 & $\mathrm{~b}$ & 1.64 & 4.90 & $\mathrm{a}$ & 4.90 & $\mathrm{a}$ & S \\
\hline Cumari Amarela do Pará A & UFGCCH 31 & 3.48 & $\mathrm{c}$ & 24582 & $\mathrm{~b}$ & 1.52 & 4.10 & $\mathrm{a}$ & 4.10 & $\mathrm{a}$ & $\mathrm{S}$ \\
\hline Amazoninha Vermelha & UFGCCH 32 & 3.63 & $\mathrm{c}$ & 9838 & $\mathrm{~b}$ & 0.85 & 4.80 & $\mathrm{a}$ & 4.80 & $\mathrm{a}$ & $\mathrm{R}$ \\
\hline Habanero Chocolate & UFGCCH 33 & 3.25 & $\mathrm{c}$ & 12283 & $\mathrm{~b}$ & 0.73 & 3.90 & a & 3.90 & $\mathrm{a}$ & $\mathrm{R}$ \\
\hline \multicolumn{12}{|l|}{ Capsicum annuum } \\
\hline Páprica 96/D & UFGCA 19 & 3.81 & $\mathrm{c}$ & 45471 & a & 3.87 & 4.00 & a & 4.00 & a & $\mathrm{S}$ \\
\hline Cayenne R3 & UFGCA 20 & 6.27 & $\mathrm{a}$ & 16902 & $\mathrm{~b}$ & 2.63 & 5.00 & a & 5.00 & $\mathrm{a}$ & S \\
\hline Indiana B & UFGCA 21 & 3.43 & $\mathrm{c}$ & 25561 & $\mathrm{a}$ & 2.01 & 4.50 & $\mathrm{a}$ & 4.50 & $\mathrm{a}$ & $\mathrm{S}$ \\
\hline Peperone & UFGCA 22 & 4.45 & $\mathrm{~b}$ & 18514 & $\mathrm{~b}$ & 1.77 & 4.50 & a & 4.50 & $\mathrm{a}$ & $\mathrm{S}$ \\
\hline Doce Comprida & UFGCA 23 & 2.94 & d & 28103 & $\mathrm{a}$ & 1.73 & 4.80 & $\mathrm{a}$ & 4.80 & $\mathrm{a}$ & $\mathrm{S}$ \\
\hline Páprica 96/F & UFGCA 24 & 2.61 & $\mathrm{~d}$ & 29618 & $\mathrm{a}$ & 1.54 & 4.00 & $\mathrm{a}$ & 4.00 & $\mathrm{a}$ & $\mathrm{S}$ \\
\hline Jalapeño B & UFGCA 25 & 2.95 & $\mathrm{~d}$ & 25551 & $\mathrm{a}$ & 1.34 & 3.80 & $\mathrm{a}$ & 3.80 & $\mathrm{a}$ & S \\
\hline La Bombonera B & UFGCA 26 & 1.91 & $\mathrm{~d}$ & 38128 & $\mathrm{a}$ & 1.30 & 3.90 & $\mathrm{a}$ & 3.90 & $\mathrm{a}$ & S \\
\hline Doce iIaliana & UFGCA 27 & 2.45 & $\mathrm{~d}$ & 15134 & $\mathrm{~b}$ & 0.75 & 3.80 & $\mathrm{a}$ & 3.80 & $\mathrm{a}$ & $\mathrm{R}$ \\
\hline Páprica 181/F & UFGCA 28 & 3.46 & $\mathrm{c}$ & 16405 & $\mathrm{~b}$ & 0.62 & 2.50 & a & 2.50 & $\mathrm{a}$ & $\mathrm{R}$ \\
\hline \multicolumn{12}{|l|}{ Capsicum baccatum } \\
\hline Fidalga Amarela B & UFGCBA 07 & 2.41 & d & 9202 & $\mathrm{~b}$ & 0.60 & 5.00 & $\mathrm{a}$ & 5.00 & $\mathrm{a}$ & $\mathrm{R}$ \\
\hline CV $(\%)$ & & 16.02 & & 43.18 & & 29.01 & 19.25 & & 19.25 & & \\
\hline
\end{tabular}

Note. Means followed by the same letter in the columns do not differ (Scott-Knot, 5\%). ${ }^{1}$ Root fresh mass; ${ }^{2}$ Population density (especimens/10 g of roots); ${ }^{3}$ Reproduction Factor; ${ }^{4}$ Gall Index; ${ }^{5}$ Egg mass index; ${ }^{6}$ Reaction. Data were transformed in $(x+1)^{1 / 2}$ for statiscal analisys.

The reproduction factor (RF) of $M$. enterolobii in susceptible genotypes ranged from 31.67 in genotype UFGCBA 01 (Coração de Galinha) to 1.01, in genotype UFGBA 06 (Dedo de Moça). Among the resistant genotypes, UFGCCH 24 (Bode Roxa A) presented RF of 0.08 and UFGFR 03 (Malagueta) showed RF equal to 0.87 (Table 1).

The nematode population density $(\mathrm{Pd})$ in the roots showed great variation in Experiment $\mathrm{I}$, and the statistical test separated the genotypes into five groups (Table 1). The group with the lowest population densities (averages followed by the letter "e" in Table 1) consisted of a total of 33 genotypes, not exactly those considered resistant by the Oostenbrink criteria (1966), adopted in the present study. Of these 33 genotypes, six were considered susceptible for showing RF higher than 1.0. In Experiment II, the test separated the genotypes into two groups, demonstrating a lower variation, but also, not a complete coincidence of the genotypes with lower Pd with the resistant genotypes. The variation found in the results from both experiments are believed to be inherent to the genotypes since the experiments were conducted under controlled conditions of a greenhouse.

The fresh root mass (FRM) also showed a great variation among the genotypes evaluated in Experiment I, but these differences were random, with no difference among the species C. chinensis, C. annum, $C$. baccatum and $C$. frutescens (Table 1). The genotypes UFGCA 02 (Carine Feltrin A), UFGCA 04 (Cumari Amarela do Pará B), and UFGCA 10 (Carine Feltrin B), all of the C. annum species presented FRM of less than ten grams. In Experiment II, all tested genotypes presented FRM ranging from $1.91 \mathrm{~g}$ to $6.27 \mathrm{~g}$ suggesting that there was some problem during the period of conduction of the second experiment that impaired the plant development.

The variables gall index (GI) and egg mass index (EMI) showed a great variation between genotypes in Experiment I and non-significant variation in Experiment II. These variables were not considered adequate for rating the genotypes as resistant or susceptible, following the Taylor and Sasser (1978) criteria. This because we found genotypes with low GI and EMI, that would be rated as resistant, but they were considered susceptible based on the RF according to Oostenbrink (1966) criteria. As an example, the genotype UFGCH 08 (Indiana A), which presented IG and EMI equal to 0.25 and genotype UFGFR 01 (Vaso Picante Super Precoce A), with a GI of 0.62 and EMI equal to 0.75 were considered susceptible based on the RF. On the other hand, genotypes with GI and EMI above 4.0 were considered resistant according to Oostenbrink (1966). 
The dendrogram resulting from the clustering analysis (Figure 1) allowed the separation of genotypes into two large groups, with the support of the cofenetic coefficient of $87 \%$ (Bray-Curtis), based on the quantitative variables studied, FR, Pd, EMI and GI. The 31 genotypes that behaved as resistant were grouped in the same clade, distinct from the others that showed a susceptibility reaction to M. enterolobii. The other groups formed, although they indicate proximity in the characteristics among the genotypes, did not group genotypes of the same species (C. annum, C. chinensis, C. baccatum and C. frutescens) so that there was a random distribution of these species.

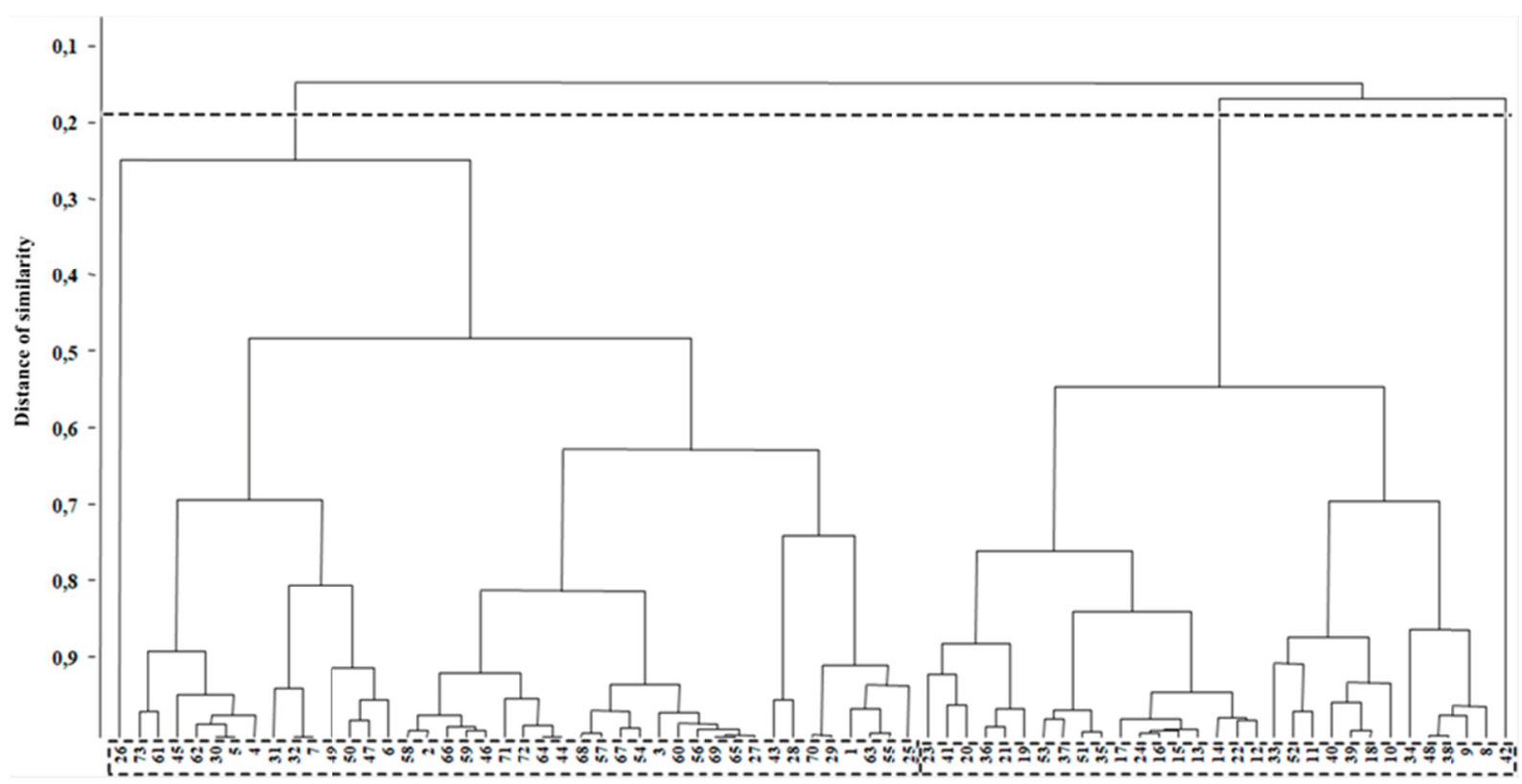

Figure 1. Dendogram from the grouping analisys of 73 genotypes of Capsicum spp. based on the population density of M. enterolobii, reproduction factor, gall index and egg mass index. Bray-Curtis-Cofenetic coeficient $=$

0.87. Genotypes: 1) Naga Jolokia A; 2) Sete Molhos; 3) Bico de Papagaio; 4) Chifre de Veado AR2; 5) Amazoninha Amarela; 6) Pimenta de Cheiro Vermelha; 7) Biquinho A; 8) Pimenta de Cheiro Amarela; 9) Bode Roxa B; 10) Fidalga Amarela A; 11) Bode Vermelha B; 12) Bode Vermelha A; 13) Habanero Amarela A; 14) Chifre de Veado C; 15) Redonda de Vaso Amarelo; 16) Bode Amarela; 17) Murupi; 18) Piramide de Vaso; 19) Vulcão Feltrin; 20) Espanhola; 21) Pitanga; 22) Redonda de Vaso Amarela; 23) Cheiro de Baiana; 24) Bode Roxa A; 25) Paprika 96L. 26) Carine Feltrin A; 27) Cumari Passarinho; 28) Cumari Amarela do Pará B; 29) Cumari B; 30) Paprika 541F; 31) Jalapeno A; 32) Indiana A; 33) Cumari A; 34) Carine Feltrin B; 35) Cumari Cheirosa; 36) Cumari Vermelha; 37) Paprika Queen; 38) Cayene; 39) La Bombonera A; 40) Pimenta Comprida; 41) Cumari C; 42) Cumari D; 43) Coração de Galinha; 44) Dedo de Moça Vermelha; 45) Cambuci; 46) Tabasco; 47) Dedo de Moça Amarela; 48) Dedo de Moça; 49) Vaso Picante Super Precoce A; 50) Malaguetinha; 51) Malagueta; 52) Malaguetão; 53) Vaso Picante Super Precoce B; 54) Naga Jolokia B; 55) Biquinho B; 56) Bode Vermelho C; 57) Chifre de Veado C; 58) Habanero Amarela B; 59) Cheiro Feltrin; 60) Cumari Amarela do Pará A; 61) Amazoninha Vermelha; 62) Habanero Chocolate; 63) Paprika 96/D; 64) Cayene R3; 65) Indiana B; 66) Peperone; 67) Doce Comprida; 68) Paprika 96/F; 69) Jalapeno B; 70) La Bombonera B; 71) Doce Italiana; 72) Paprika 181/F; 73) Fidalga Amarela B

Principal components analysis based on the variables genotypes, FR, Pd, GI and EMI explained $76.75 \%$ of the total variability, and $51.52 \%$ of the variance was explained by the main component 1 (PC1) and $25.23 \%$ by the main component 2 (PC2) (Figure 2). The genotypes grouped in the upper and lower right quadrants are considered susceptible because they are more correlated with the vectors representing FR and Pd (upper quadrant) and GI and EMI (lower quadrant). The resistant genotypes separated according to the variables EMI and GI are represented in the upper left quadrant and the resistant ones with low values of FR and Pd in the lower left quadrant. 


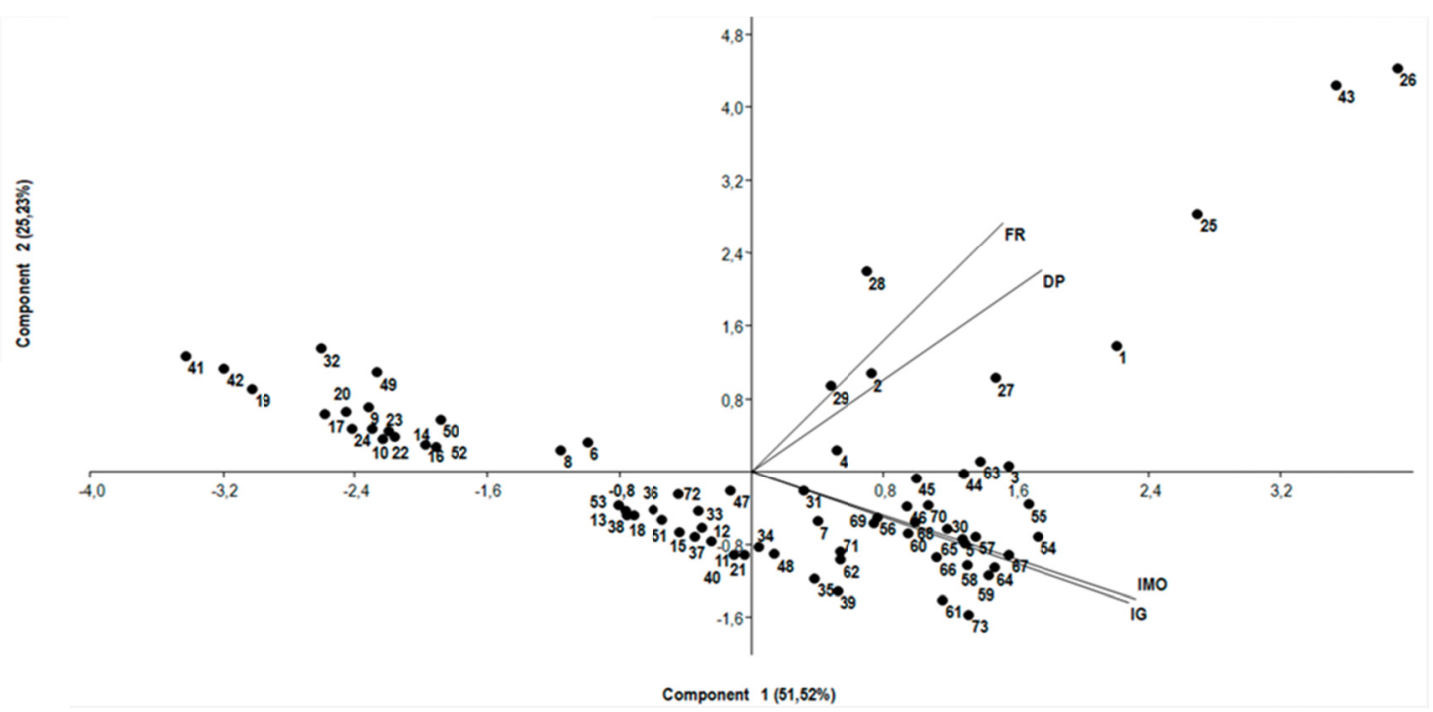

Figure 2. Biplot of variables referring to resistance/susceptibility to the nematode Meloidogyne enterolobii and genotypes of Capsicum spp. related to principal components 1 and 2. RF: Reproduction Fator; Pd: Population density; EMI: Egg mass index; GI: Gall index. Genotypes: 1) Naga Jolokia A; 2) Sete Molhos; 3) Bico de Papagaio; 4) Chifre de Veado AR2; 5) Amazoninha Amarela; 6) Pimenta de Cheiro Vermelha; 7) Biquinho A; 8) Pimenta de Cheiro Amarela; 9) Bode Roxa B; 10) Fidalga Amarela A; 11) Bode Vermelha B; 12) Bode Vermelha A; 13) Habanero Amarela A; 14) Chifre de Veado C; 15) Redonda de Vaso Amarelo; 16) Bode Amarela; 17) Murupi; 18) Piramide de Vaso; 19) Vulcão Feltrin; 20) Espanhola; 21) Pitanga; 22) Redonda de Vaso Amarela;

23) Cheiro de Baiana; 24) Bode Roxa A; 25) Paprika 96L; 26) Carine Feltrin A; 27) Cumari Passarinho; 28) Cumari Amarela Pará B; 29) Cumari B; 30) Paprika 541F; 31) Jalapeño A; 32) Indiana A; 33) Cumari A; 34)

Carine Feltrin B; 35) Cumari Cheirosa; 36) Cumari Vermelha; 37) Paprika Queen; 38) Cayenne; 39) La Bombonera A; 40) Pimenta Comprida; 41) Cumari C; 42) Cumari D; 43) Coração de Galinha; 44) Dedo de Moça Vermelha; 45) Cambuci; 46) Tabasco; 47) Dedo de Moça Amarela; 48) Dedo de Moça; 49) Vaso Picante Super Precoce A; 50) Malaguetinha; 51) Malagueta; 52) Malaguetão; 53) Vaso Picante Super Precoce B; 54) Naga Jolokia B; 55) Biquinho B; 56) Bode Vermelho C; 57) Chifre de Veado C; 58) Habanero Amarela B; 59) Cheiro Feltrin; 60) Cumari Amarela do Pará A; 61) Amazoninha Vermelha; 62) Habanero Chocolate; 63) Paprika 96/D; 64) Cayenne R3; 65) Indiana B; 66) Peperone; 67) Doce Comprida; 68) Paprika 96/F; 69) Jalapeño B; 70) La Bombonera B; 71) Doce Italiana; 72) Paprika 181/F; 73) Fidalga Amarela B

The genotypes UFGCH 02 (Sete Molhos) and UFGCBA 03 (Cambuci) presented RF of M. enterolobii greater than 1.0 at all inoculum concentrations tested (Table 2), which was already expected, since they were used as standard of susceptibility based on the results of Experiment I. The other evaluated genotypes, that were resistant in Experiment I, remained resistant even under increasing inoculum concentrations. 
Table 2. Reproduction fator of $M$. enterolobii in sixteen genotypes of Capsicum spp. subjected to inoculum concentrations of de 2000, 4000 and 8000 eggs $+\mathrm{J} 2$ per plant, at 60 days after inoculation. UFG, GO, 2017

\begin{tabular}{|c|c|c|c|c|c|c|c|}
\hline \multirow{3}{*}{$\begin{array}{l}\text { Genotypes } \\
\text { Sete Molhos }\end{array}$} & \multicolumn{7}{|c|}{ Reproduction Factor (RF) } \\
\hline & \multirow{2}{*}{$\begin{array}{l}\text { ID UFG } \\
\text { UFGCCH } 02\end{array}$} & \multicolumn{2}{|c|}{2000} & \multicolumn{2}{|c|}{4000} & \multicolumn{2}{|l|}{8000} \\
\hline & & 1.74 & $\mathrm{a}$ & 1.22 & $\mathrm{a}$ & 1.05 & $\mathrm{a}$ \\
\hline Cambuci & UFGCBA 03 & 1.26 & a & 1.43 & a & 1.24 & a \\
\hline Cayenne R3 & UFGCA 14 & 0.22 & $\mathrm{~b}$ & 0.09 & $\mathrm{c}$ & 0.28 & $\mathrm{~b}$ \\
\hline Vaso Picante Super Precoce B & UFGFR 05 & 0.21 & $\mathrm{~b}$ & 0.36 & $\mathrm{~b}$ & 0.04 & $\mathrm{c}$ \\
\hline Páprica Queen & UFGCA 13 & 0.20 & $\mathrm{~b}$ & 0.06 & $\mathrm{c}$ & 0.43 & $\mathrm{~b}$ \\
\hline Vulcão Feltrin & UFGCCH 19 & 0.19 & $\mathrm{~b}$ & 0.08 & $\mathrm{c}$ & 0.16 & $\mathrm{~b}$ \\
\hline Pitanga & UFGCCH 21 & 0.17 & $\mathrm{~b}$ & 0.09 & $\mathrm{c}$ & 0.03 & $\mathrm{c}$ \\
\hline Bode Roxa A & UFGCCH 24 & 0.16 & $\mathrm{~b}$ & 0.31 & $\mathrm{~b}$ & 0.03 & $\mathrm{c}$ \\
\hline La Bombonera A & UFGCA 15 & 0.14 & $\mathrm{~b}$ & 0.15 & $\mathrm{~b}$ & 0.04 & $\mathrm{c}$ \\
\hline Malagueta & UFGFR 03 & 0.14 & $\mathrm{~b}$ & 0.01 & $\mathrm{c}$ & 0.05 & $\mathrm{c}$ \\
\hline Redonda de Vaso Amarela & UFGCCH 22 & 0.13 & $\mathrm{~b}$ & 0.08 & $\mathrm{c}$ & 0.04 & $\mathrm{c}$ \\
\hline Cumari Vermelha & UFGCA 12 & 0.11 & $\mathrm{~b}$ & 0.07 & $\mathrm{c}$ & 0.38 & $\mathrm{~b}$ \\
\hline Pirâmide de Vaso & UFGCCH 18 & 0.10 & $\mathrm{~b}$ & 0.13 & $\mathrm{~b}$ & 0.03 & $\mathrm{c}$ \\
\hline Espanhola & UFGCCH 20 & 0.09 & $\mathrm{c}$ & 0.02 & $\mathrm{c}$ & 0.11 & $\mathrm{~b}$ \\
\hline Malaguetão & UFGFR 04 & 0.08 & $\mathrm{c}$ & 0.08 & $\mathrm{c}$ & 0.02 & $\mathrm{c}$ \\
\hline Chifre de Veado C & UFGCCH 28 & 0.03 & c & 0.05 & $\mathrm{c}$ & 0.23 & $\mathrm{~b}$ \\
\hline CV $(\%)$ & 4.73 & & & & & & \\
\hline
\end{tabular}

Note. Means followed by the same letter do not differ (Scott-Knott, 5\%). Data were transformed in $\sqrt{(\mathrm{x}+1)}$ for the statistical analisys.

The susceptible genotypes UFGCCH 02 (Sete Molhos) and UFGCBA 03 (Cambuci) presented the highest population densities (Pd) of $M$. enterolobii. The curves representing the regression equations for these two genotypes are of a quadratic nature, showing that the nematode population densities in the roots increased as the inoculum concentration increased to a maximum of 5344 eggs $+\mathrm{J} 2 / 10 \mathrm{~g}$ of root, for Sete Molhos, and 7781 eggs $+\mathrm{J} 2 / 10 \mathrm{~g}$ of root for Cambuci. With respect to the other genotypes, only seven presented a significant regression analysis. UFGCA 20 (Cayenne R3) and UFGCA 13 (Paprika Queen) presented linear regression with increase of $\mathrm{Pd}$ as the inoculum concentration was increased. The genotypes UFGFR 05 (Vaso Picante Super Precoce B) and UFGCH 24 (Bode Roxa A) presented a small increase of Pd decreasing again as the inoculum concentration increased, while the genotypes UFGCA 14 (Cayenne R3), UFGCA 13 (Paprika Queen), UFGCA 12 (Cumari Vermelha), UFGCH 19 (Vulcão Feltrin) and UFGCH 28 (Chifre de Veado C) had opposite behavior, with reduction and subsequent increase of Pd with increasing inoculum concentration (Figure 3).

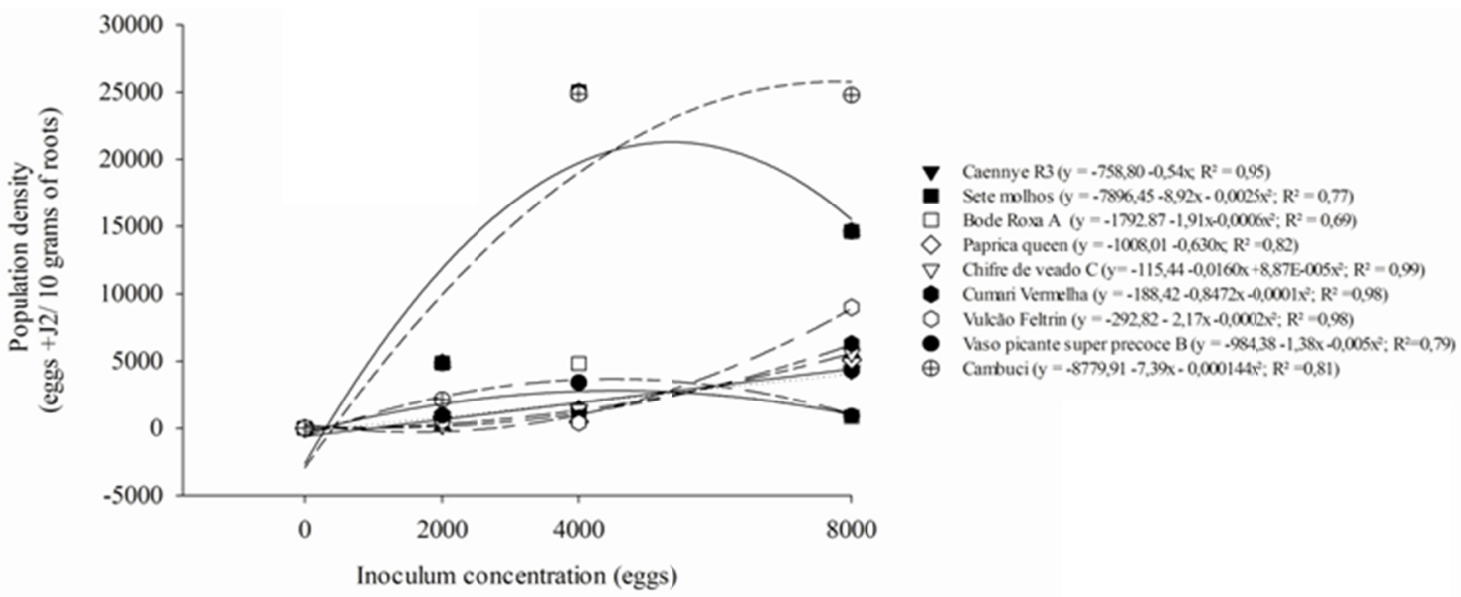

Figure 3. Population density of Meloidogyne enterolobii in $10 \mathrm{~g}$ of roots of nine Capsicum spp. genotypes subjected to different inoculum concentrations of Meloidogyne enterolobii at 60 days after inoculation 


\section{Discussion}

31 pepper genotypes resistant to M. enterolobii were identified (Table 1). The genotypes of Capsicum spp. used in the present study belong to the collection of chili peppers of the UFG, and were first tested for hostability to $M$. enterolobii. Resistance reactions found in the present study are unprecedented, since to date, there are no reports of resistance sources to this nematode in Capsicum spp., although Melo et al. (2011) found moderate levels of resistance in two accessions of chili pepper and three accessions of sweet pepper. However, these authors used the Taylor (1967) criteria for classification of genotypes, so that only one access of sweet pepper showed RF less than 1.0.

It is important to note that within each of the four species of Capsicum (C. chinenses, $C$. annuum, $C$. baccatum and $C$. frutescens) tested, resistant genotypes were found, which suggests the presence of resistance genes within the whole complex. It is known that domesticated species of Capsicum spp., usually are autogamous, however, there is a possible rate of allogamy that can vary from 0 to $83 \%$, being facilitated by morphological changes in the flower by the action of pollinating insects, and other factors (Moreira et al., 2006), thus generating a high rate of genetic variability among the genotypes. This suggests that sources of nematode resistance can be identified within a large set of genes in the Capsicum complex. On the other hand, it is possible that among the genotypes studied here, seeds of the same genotype have been identified as being of different genotypes, which may have led to the high number of resistant materials. This may have occurred since the separation and identification of the genotypes was made based on the fruit traits. But, still, there are a considerable number of resistant genotypes.

In the dendrogram resulting from genotype cluster analysis (Figure 1), which took into consideration, in addition to the RF, the population density, gall index and egg mass index, there was a clear separation of the resistant and susceptible genotypes, confirming the genotype ratings presented in Table 1. These characteristics, however, did not group genotypes of the same species of Capsicum so that the species were distributed randomly, strengthening the hypothesis that resistance sources are present in the four species of Capsicum studied.

There are several reports of resistance reaction of Capsicum spp. to M. javanica and M. incognita (Oliveira et al., 2009, Pinheiro et al., 2013, Pinheiro et al., 2014). But most studies on Capsicum spp. genotypes behavior in relation to M. enterolobii resulted in susceptibility reactions such as Pinheiro et al. (2014), that evaluated Capsicum spp. genotypes against the infestation by $M$. enterolobii and all reacted as susceptible. Reaction of susceptibility was also reported by Melo et al. (2011) in accessions of $C$. chinenses and C. annum. Rosa et al. (2015) found susceptibility reactions in Jalapeño, Dedo de Moça and Cambuci, which was also observed in the present study. However, these same authors observed a susceptibility reaction in the Doce Italiana and Malagueta genotypes, which proved to be resistant in our study.

Resistance and susceptibility according to the criterion of Oostenbrink (1966) shows how well the nematode may reproduce on the plant genotypes. In this way, genotypes that show RF lower than 1.0 are considered resistant. Those with the RF higher than 1.0 are considered susceptible. Although it is very usual to find variable results between experiments when working with nematodes the variation found in this study seems to be related to the differences among the genotypes. Experiments I and II tested different Capsicum spp. genotypes and complemented each other.

Principal components analysis showed a high correlation between the nematode RF and Pd and these variables were inversely related to the GI and EMI (Figure 2). The genotypes that reacted with susceptibility with high EMI and GI were grouped and were arranged in the lower right quadrant of the graph. Likewise, susceptible genotypes with high RF and Pd values were grouped in the upper right quadrant. Resistant genotypes were grouped in the lower and upper left quadrants and correlated inversely with the FR, Pd, EMI and GI.

Increasing inoculum levels of $M$. enterolobii did not shift the resistance or susceptibility behavior of the tested genotypes, considering the RF (Table 2). In relation to the population density (Pd), the resistant genotypes presented values far below of those observed in the two standard susceptible genotypes at all inoculum concentrations (Figure 3). It was observed that the susceptible genotypes UFGCH 02 (Sete Molhos) and UFGCBA 03 (Cambuci) showed maximum nematode development in the roots (Pd) within the tested inoculum concentration range, decreasing again, indicating that at very high doses there may be competition, reducing the efficiency of inoculation. Among the resistant genotypes, UFGFR 05 (Vaso Picante Super Precoce B) and UFGCH 24 (Bode Roxa A) are considered the most promising because they showed increase in Pd as the inoculum concentration was increased, decreasing again, and were those that presented the lowest $\mathrm{Pd}$ of $M$. enterolobii in the highest concentration (8000 eggs $+\mathrm{J} 2 /$ plant) (Figure 3$)$. 
The results obtained here suggest that the resistant genotypes may serve as the object of studies in the identification of promising genes for the chili peppers genetic breeding. In this way, the present study represents a relevant contribution with the identification of Capsicum spp. genotypes resistant to M. enterolobii that can be used by growers in areas infested by this plant parasitic nematode. In addition, these genotypes can be used as rootstocks for other Solanaceae, susceptible to this nematode, such as sweet pepper, since there is compatibility. Further studies are needed to identify the genes and the mechanisms involved in the resistance present in these genotypes so that they can be used in breeding programs to develop new cultivars resistant to M. enterolobii.

\section{References}

Bitencourt, N. V., \& Silva, G. S. (2010). Reprodução de Meloidogyne enterolobii em olerícolas. Nematologia Brasileira, 34, 181-183.

Bonetti, J. I. S., \& Ferraz, S. (1981). Modificações do método de Hussey e Barker para extração de ovos de Meloidogyne exigua em raízes de cafeeiro. Fitopatologia Brasileira, 6, 553.

Carneiro, R. G., Mônaco, A. P. A., Moritz, M. P., Nakamura, K. C., \& Scherer, A. (2006). Identificação de Meloidogyne mayaguensis em goiabeira e em plantas invasoras, em solo argiloso, no Estado do Paraná. Nematologia Brasileira, 30, 293-298.

Carneiro, R. M. D. G., Moreira, W. A., \& Gomes, A. C. M. M. (2001). Primeiro registro de Meloidogyne enterolobii em goiabeira no Brasil. Nematologia Brasileira, 25, 223-228.

Carneiro, R. M. D., \& Almeida, M. R. A. (2001). Técnica de eletroforese usada no estudo de enzimas dos nematoides de galhas para identificação de espécie. Nematologia Brasileira, 2, 35-44.

Conagin, A., Nagqai, V., Igue, T., \& Ambrósio, L. A. (1993). Efeito da falta de normalidade em testes de homogeneidade de variâncias. Bragantia, 52, 173-180. https://doi.org/10.1590/S0006-87051993000200010

Coolen, W. A., \& D'Herde, C. J. A. (1972). A method for the quantitative extraction of nematodes from plant tissue. Ghent, Belgium: State Nematology and Entomology Research Centre.

Costa, C. S. R., \& Henz, G. P. (2007). Pimentas Capsicum spp. Botânica. Sistema de Produção 2. Brasilía: Embrapa hortaliças.

Fargette, M. (1987). Use of esterase phenotype in the taxonomy of the genus Meloidogyne. 2. Esterase phenotypes observed in West African populations and their characterization. Revue de Nématologie, 10, $45-56$.

Ferreira, D. F. (2011). Sisvar: A computer statistical analysis system. Ciência e Agrotecnologia, 35, 1039-1042. https://doi.org/10.1590/S1413-70542011000600001

Hair Junior, J. F., Black, W. C., Babin, B. J., Anderson, R. E., \& Tatham, R. L. (2005). Análise multivariada de dados. Porto Alegre, Brazil: Artmed.

Hammer, Ø., Harper, D. A. T., \& Ryan, P. D. (2001). PAST: Software de estatística paleontológica para educação e análise de dados. Palaeontologia Electronica, 4, 1-9.

Henz, G. P., \& Ribeiro, C. S. C. (2008). Mercado e comercialização. In C. S. C. Ribeiro, C. A. Lopes, S. I. C. Carvalho, G. P. Henz, \& F. J. B. Reifschneider (Eds.), Pimentas Capsicum (pp. 15-24). Brasília, Brazil: Embrapa Hortaliças.

Hussey, R. S., \& Barker, K. R. (1973). A comparison of methods of collection inocula of Meloidogyne spp. including a new technique. Plant Desease Reporter, 57, 1025-1028.

Melo, O. D., Maluf, W. R., Gonçalves, R. J. S., Gonçalves Neto, A. C., Gomes, L. A. A., \& Carvalho, R. C. (2011). Triagem de genótipos de hortaliças para resistência a Meloidogyne enterolobii. Pesquisa Agropecuária Brasileira, 46, 829-835. https://doi.org/10.1590/S0100-204X2011000800007

Moreira, G. R., Caliman, F. R. B., Silva, D. J. H., \& Ribeiro, C. S. C. (2006). Espécies e variedades de pimenta. Informe Agropecuário, 27, 16-19.

Oliveira, C. D., Braz, L. T., Santos, J. M., Banzatto, D. A., \& Oliveira, P. R. (2009). Resistência de pimentas a nematoides de galha e compatibilidade enxerto/porta-enxerto entre híbridos de pimentão e pimentas. Horticultura Brasileira, 27, 520-526. https://doi.org/10.1590/S0102-05362009000400019

Oostenbrink, M. (1966), Major characteristics of the relation between nematodes and plants. Mededelingen Landbouwhogoe School, 66, 1-46. 
Pinheiro, J. B., Reifschneider, F. J. B., Pereira, R. B., \& Moita, A. W. (2013). Reprodução de Meloidogyne spp. em Capsicum spp. Nematologia Brasileira, 37, 20-25.

Pinheiro, J. B., Reifschneider, F. J. B., Pereira, R. B., \& Moita, A. W. (2014). Reação de genótipos de Capsicum ao nematoide-das-galhas. Horticultura Brasileira, 32, 371-375. https://doi.org/10.1590/S0102-0536201400 0300022

Reifschneider, F. J. B. (2000). Capsicum: Pimentas e pimentões no Brasil. Brasília, Brazil, Embrapa Hortaliças.

Ribeiro, C. S. C., Lopes, C. A., Carvalho, S. I. C., \& Reifschneider, F. J. B. (2008). Pimentas Capsicum spp. Brasília, Brazil, Embrapa Hortaliças.

Rosa, J. M. O., Westerich, J. N., \& Wilcken, S. R. S. (2015). Reprodução de Meloidogyne enterolobii em olerícolas e plantas utilizadas na adubação verde. Revista Ciência Agronômica, 46, 826-835. https://doi.org/ 10.5935/1806-6690.20150071

Rufino, J. L. S., Penteado, D. C. S. (2006). Importância econômica, perspectivas e potencialidades do mercado para pimenta. Informe Agropecuário, 27, 7-15.

Sasser, J. N., \& Kirby, M. F. (1979). Crop cultivars resistant to root-knot nematodes, Meloidogyne species, with information on seed sources. Raleigh, USA: North Carolina State University.

Silva, F. A. S. (2017). Assistat: Versão 7.7beta. Retrieved from http://www.assistat.com

Taylor, A. L. (1967). Introduction to research on plant nematology: An FAO guide to the study and control of the plant-parasitic nematodes. Rome, Italy: Food and Agricultural Organization of the United Nations.

Taylor, A. L., \& Sasser, J. N. (1978). Biology, identification and control of root-knot nematodes (Meloidogyne spp.). Raleigh, USA: North Carolina State University.

\section{Copyrights}

Copyright for this article is retained by the author(s), with first publication rights granted to the journal.

This is an open-access article distributed under the terms and conditions of the Creative Commons Attribution license (http://creativecommons.org/licenses/by/4.0/). 